\title{
DISENO E IMPLEMENTACION DE UN MODULO DE GESTION DE ENERGIA PARA
UN PICO-SATELITE TIPO CUBESAT
} JORGE ENRIQUE SALAMANCA CÉSPEDES and ROBERTO FERRO ESCOBAR

Universidad Distrital Francisco José de Caldas,Bogotá, Colombia

\section{Abstract}

This article briefly describes the development of Power Module for Experimental picosatellite CubeSat UD Colombia 1 following CubeSat standard requirements. Whether the Power Module project consists of four stages of development: study, design, implementation and testing. In the study phase to review the theoretical framework and preliminary designs made in the Universidad Distrital and other Cubesat developed in the world, also investigates existing components and

Corresponding Author: JORGE ENRIQUE SALAMANCA CÉSPEDES

jesalamancac@gmail.com

Received: 15 November 2017

Accepted: 5 January 2018

Published: 4 February 2018

Publishing services provided by Knowledge $\mathrm{E}$

(c) JORGE ENRIQUE

SALAMANCA CÉSPEDES and ROBERTO FERRO

ESCOBAR. This article is distributed under the terms of the Creative Commons

Attribution License, which permits unrestricted use and redistribution provided that the original author and source are credited.

Selection and Peer-review under the responsibility of the ESTEC Conference Committee.

G OPEN ACCESS technologies in the market. The design phase involves analysis of the system and using a computer program designed to generate the necessary hardware. The implementation consists in making the printed circuit board and the component assembly. And electrical type tests to certify the proper operation of the module. The development of the power module of the CubeSat standard requirements and mission picosatellite, and depends on the state and information available from other modules picosatellite. The ultimate goal is to obtain a power module that is functional and working conditions of the space environment in which the picosatellite fulfill its focused on telemedicine, with a payload that would become the telecommunications system mission.

Keywords: Power Module, CubeSat UD Colombia 1, Standard CubeSat, DC-DC converters, Solar Panels, Batteries, Power Management.

\section{Resumen}

Este artículo trata sobre el desarrollo del Módulo de Potencia para el picosatelite experimental CubeSat UD Colombia 1 siguiendo el estándar de requerimientos CubeSat. En si el proyecto del Módulo de Potencia se desarrolló en cuatro fases: estudio, diseño, implementación y pruebas. En la fase de estudio, se revisa el marco teórico y los diseños preliminares hechos en la Universidad Distrital y de otros CubeSat desarrollados en el mundo, también investiga tecnologías y componentes existentes en el mercado. La fase de diseño implica el análisis del sistema y la utilización de un programa de 
diseño computacional para generar el hardware necesario. La implementación consiste en la fabricación de la tarjeta de circuito impreso y el ensamble de componentes. Y las pruebas de tipo eléctrico para certificar el buen funcionamiento del módulo. El desarrollo del Módulo de Potencia parte de los requerimientos del estándar CubeSat y de la misión del picosatelite, del ambiente espacial y depende del estado y la información disponible de otros módulos del picosatelite. El objetivo final es obtener un Módulo de Potencia que sea funcional y que trabaje las condiciones del entorno espacial en el que el picosatelite cumplirá su misión enfocada a telemedicina, con una carga útil que vendría a ser el sistema de telecomunicaciones.

Palabras claves: Módulo de Potencia, CubeSat UD Colombia 1, Estándar CubeSat, Convertidores DC-DC, Paneles Solares, Baterías, Gestión de Energía.

\section{INTRODUCCIÓN}

El Módulo de Potencia es un elemento clave en cualquier satélite, su función es la de asegurar el constante suministro de energía eléctrica para el funcionamiento de los distintos subsistemas del satélite en el espacio exterior. Hace uso como en la mayoría de satélites de una fuente de energía "inagotable" en el espacio exterior, ésta es la radiación solar, constituyéndose en la principal fuente de energía, energía que se puede aprovechar para convertirla en otras formas de energía como la eléctrica. Y esto lo hace a través de paneles solares.

Los paneles solares transforman la energía solar en energía eléctrica usando celdas fotovoltaicas. La energía eléctrica obtenida es entregada al Módulo de Potencia el cual la acondiciona, la transfiere y la distribuye a los distintos usuarios del picosatélite de acuerdo a las necesidades de consumo de estos, los satélites generalmente se diseñan para evitar la falta de energía durante eclipses (esto ocurre al obstruir la tierra, la radiación solar hacia el satélite) o como complemento a requerimientos de consumo, se requiere contar con un sistema de almacenamiento de energía, es decir baterías, ver figura 1.

Hay que tener presente que no todos los sistemas en un satélite trabajan todo el tiempo, ni al mismo tiempo, por la tanto hay que gestionar, y administrar la energía para optimizarla dentro de los límites de tolerancia y niveles posibles para alargar la vida útil del satélite. 
El Módulo de Potencia asegurar los niveles de voltaje y corriente requeridos por los diferentes módulos del satélite hasta en los escenarios de operación más críticos y en las condiciones más adversas. También generalmente estos subsistemas trabajan con potencia $\mathrm{DC}$, y lo que se hace es una conversión con reguladores DC/DC, reductores y/o elevadores y que vienen a constituirse en la unidad central del Módulo de Potencia.

Por otro lado teniendo en cuenta que el espacio exterior es el medio en que trabaja el satélite. El Módulo de Potencia debe prevenir fallos y tomar decisiones si ocurren eventos críticos y por lo tanto la tecnología utilizada debe tener características especiales, diferentes a los elementos utilizados en la Tierra. Por eso hay que investigar las tecnologías disponibles en el mercado.

Este proyecto desarrolla un módulo funcional de suministro de potencia para el picosatélite experimental CubeSat UD Colombia 1 que funcione en las condiciones del espacio exterior.

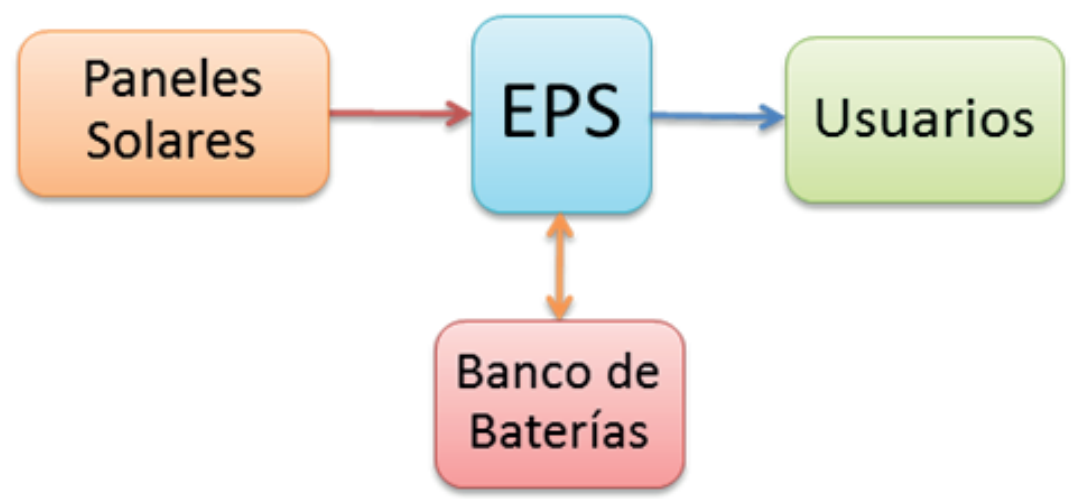

Figura 1: Configuración Básica del Módulo de Potencia

El satélite será lanzado en la "Órbita Terrestre Baja" (LEO Low Eorth Orbit) siguiendo una trayectoria Polar a una velocidad cercana a los $27000 \mathrm{Km} / \mathrm{h}$, con un periodo orbital de entre 90 minutos y 110 minutos, esto es, hace de entre 13 y 16 orbitas por día. La altura del picosatélite está entre los $600 \mathrm{Km}$ y los 800 km. Esa órbita baja permite que la potencia necesaria para transmisión y recepción de información sea menor en comparación con las orbitas superiores, algo acorde al tamaño, peso y enfoque académico del picosatélite.

En el espacio existen básicamente dos tipos de radiación que afectan el satélite. La radiación electromagnética y la radiación de partículas. La mayor parte de esta radiación proviene directamente del sol, pero la radiación cósmica y la radiación de partículas desde el campo magnético de la Tierra también son significativas. Ambos 
tipos de radiación, de partículas y electromagnética, degradan con el tiempo la electrónica. Por esa razón los dispositivos electrónicos usados ??a bordo tienen unas características y rangos especiales de funcionamiento y han de ser probados en tierra para asegurarse de que son lo suficientemente resistentes para soportar la radiación.

A una altitud de 600 kilómetros hay muy poca atmosfera y se puede considerar un "vacío". Esto implica que los componentes que consisten en materiales volátiles sufrirán de evaporación (desgasificación) y por lo tanto todos los componentes utilizados en la fuente de alimentación deben ser probados a fondo por su capacidad para soportar el vacío, por esta razón todo el transporte de calor será por conducción o por radiación, pero nunca convección. Debido a esto, se debe considerar que los problemas pueden surgir con respecto a la descarga de calor desde el satélite, por lo que la fuente de alimentación debe estar construida de manera que la producción de calor sea tan baja como sea posible. El rango de temperatura que el satélite debe ser capaz de soportar durante el lanzamiento es de $-40^{\circ} \mathrm{C}$ a $80^{\circ} \mathrm{C}$, estas son las temperaturas a las que el satélite está expuesto cuando está en el vehículo lanzador (cohete).

Las temperaturas en órbita dependerán principalmente del diseño térmico del satélite y como no se ha realizado para esta misión, se dependerá de los cálculos de otras universidades que han construido satélites CubeSat. El TIT de Tokio calcula que las temperaturas en órbita deben varía de $-40^{\circ} \mathrm{C} \mathrm{a} 80^{\circ} \mathrm{C}$ y la SSEL de Montana estima que el intervalo de temperatura es de $-120^{\circ} \mathrm{C}$ a $100^{\circ} \mathrm{C}$ externamente. Aunque hay diferencias en los cálculos de las dos universidades se puede concluir que el satélite debe ser capaz de sobrevivir en un ambiente térmico muy hostil, con temperaturas exteriores que cambian bruscamente, sometiendo al satélite a 'estrés' térmico.

Siempre y cuando el satélite está en órbita, no habrá vibraciones inducidas externamente, y la aceleración será menor que en la tierra. Durante el lanzamiento, sin embargo, habrá fuertes vibraciones y una potente aceleración. El satélite, y por ende el suministro de alimentación de la placa, tiene que ser capaz de soportar una aceleración de $15 \mathrm{~g}$.

\section{FUNCIONES DEL MODULO DEL POTENCIA}

La EPS debe cumplir como mínimo con las siguientes funciones:

- Proveer una fuente continua de la corriente eléctrica a las cargas de la nave espacial durante la vida de la misión. 
- Controlar y distribuir la corriente eléctrica a la nave espacial.

- Cumplir los requerimientos de potencia eléctrica promedio y pico de los usuarios.

- Proporcionar líneas de bus reguladas en potencia DC.

- Proporcionar capacidad de comando y de telemetría para la integridad y estado de la EPS, así como el control desde la estación terrena o del sistema autónomo.

- Proteger la carga útil de la nave espacial contra fallas dentro del Sistema de Potencia Eléctrica.

- Suprimir los voltajes transitorios en las líneas de bus reguladas y protegerlos contra averías.

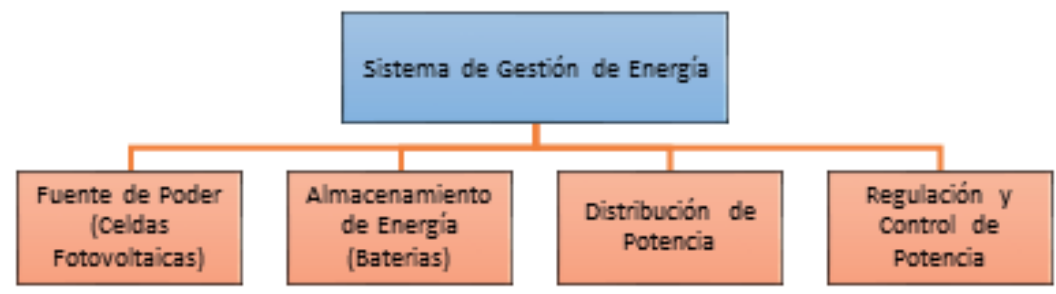

Figura 2: Elementos de un Módulo de Potencia.

\subsection{Requerimientos De Consumo Potencia Del Picosatélite}

Para determinar los alcances máximos la EPS se ha determinado unos valores próximos máximos o picos de consumo de los diferentes subsistemas del picosatélite que son el Módulo de Comunicaciones (COMM), el Modulo de Computador a Bordo (OBC), el Modulo de Actitud (ATT) y el propio Modulo de Potencia (EPS) en base a información suministrada por otros Módulos o estimativos en base a otros picosatélites en el mundo.

La tabla 1 muestra esos valores de consumo límites extremos, en funcionamiento normal esos valores son más bajos, y en cuanto al tiempo de consumo no están sostenidos en el tiempo, sino en breves periodos, dependiendo del subsistema. Se hace una gestión de la energía asociada a cada módulo del satélite de acuerdo al momento del requerimiento de energía.

\subsection{Paneles Solares}

El grupo de trabajo del Módulo de Paneles Solares (AGUILAR PIRACHICÁN Clara Milena y AMAYA CAMARGO Andrés Augusto. Estudio del Sistema de Celdas Fotovoltaicas para la 
TABLA 1: Presupuesto de energía (consumo pico) subsistemas picosatelite.

\begin{tabular}{|c|c|c|c|c|c|}
\hline $\begin{array}{l}\text { Subsistema } \\
\text { picosatélite }\end{array}$ & Voltaje [V] & $\begin{array}{c}\text { Corriente } \\
{[\mathrm{mA}]}\end{array}$ & $\begin{array}{c}\text { Potencia } \\
\text { [mW] }\end{array}$ & $\begin{array}{c}\text { Tiempo de } \\
\text { operación por } \\
\text { orbita (97min), } \\
\text { [min] }\end{array}$ & $\begin{array}{l}\text { Energía } \\
\text { [mWh] }\end{array}$ \\
\hline $\mathrm{OBC}$ & 5 & 40 & 200 & 97 & 323,33 \\
\hline COMM Recepción & 5 & 70 & 350 & 58 & 338,33 \\
\hline COMM Beacon & 5 & 500 & 2500 & 29 & 1208,33 \\
\hline COMM Trasmisión & 5 & 500 & 2500 & 10 & 416,67 \\
\hline MCU's y sensores & 3,3 & 50 & 165 & 97 & 266,75 \\
\hline ATT & 5 & 200 & 1000 & 49 & 816,67 \\
\hline EPS Calentadores & 5 & 150 & 750 & 30 & 375,00 \\
\hline
\end{tabular}

Implementación de los Paneles Solares del CubeSat Colombia 1. Bogotá, 2011. Trabajo de Grado (Ingeniero Electrónico). Universidad Distrital Francisco José de Caldas. Facultad de Ingeniería. Coordinación de Ingeniería Electrónica.) proporciona el prototipo para el arreglo de celdas fotovoltaicas más conveniente para su utilización en los paneles solares del picosatélite experimental CubeSat UD Colombia 1, en base a un estudio de celdas disponibles en el mercado. El resultado de este trabajo son paneles solares no desplegables y que están montados en las caras de un picosatélite tipo CubeSat de tamaño $1 \mathrm{U}$.

El modelo utilizado por ellos para el diseño de los paneles solares se basa en fuentes de corriente ya que tiene similitudes en la forma como las celdas fotovoltaicas convierten la energía solar en energía eléctrica. El voltaje proporcionado en cada celda fotovoltaica tiende a ser estable en la salida, con variaciones en la corriente de celda que es proporcional a la energía recibida por esta. El esquemático de circuito de cada Panel Solar se muestra en la figura 3.

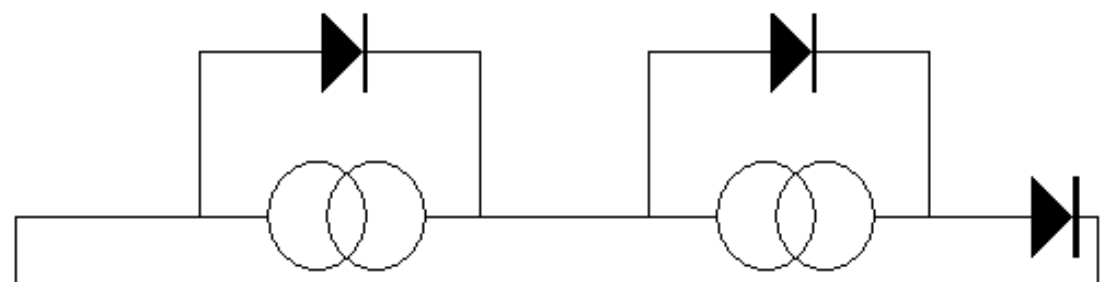

Figura 3: Configuración del panel solar para el picosatélite CubeSat UD Colombia 1. 


\section{TOPOLOGIAS BASICAS PARA LOS REGULADORES EN EL MODULO DE POTENCIA (Diseño)}

Los tres pilares fundamentales considerados para la topología de diseño de la EPS son el panel solar, la batería y el regulador/convertidor de voltaje DC-DC.

Existen diferentes topologías en base a la cantidad de conversores dc-dc utilizados y según la disposición-ubicación de los tres elementos fundamentales mostrados en la figura 4, y los requerimientos de la tabla 1.

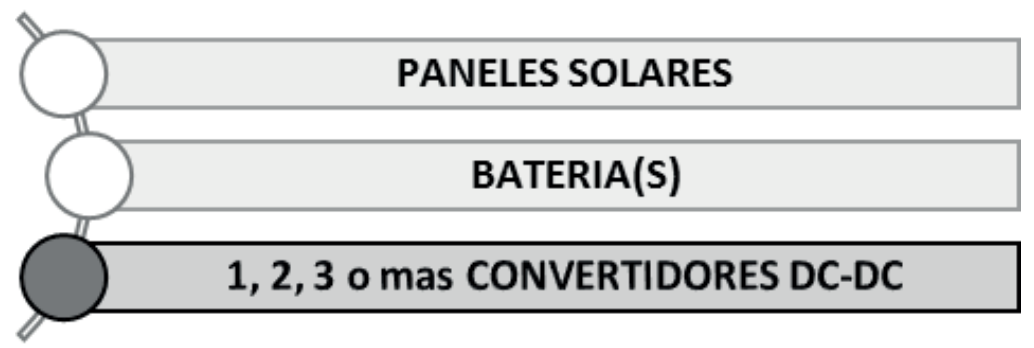

Figura 4: Elementos fundamentales vinculados al diseño de la topología de diseño de la EPS.

Para el diseño de la topología se evalúo distintos factores como espacio físico, temperatura, eficiencia en energía, máxima transferencia de potencia, capacidad de control de los circuitos, buses regulados o no, robustez del módulo, minimización de los fallos, complejidad del circuito, ruido, caminos de potencia, entre otros factores tenidos en cuenta.

Teniendo presente estos factores la topología escogida para el diseño de la EPS del picosatélite de la Universidad se muestra en la figura 5:

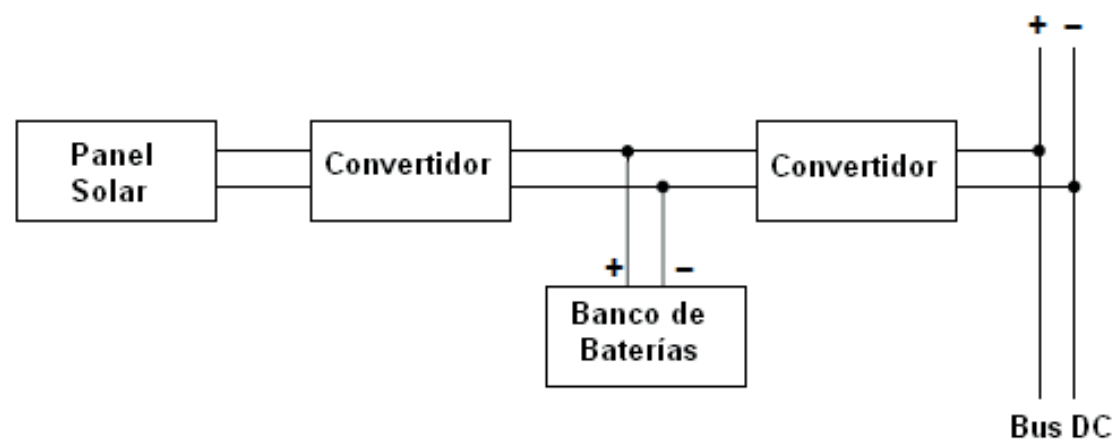

Figura 5: Topología seleccionada. 


\subsection{Convertidor DC-DC}

Un convertidor DC-DC es un circuito regulador electrónico capaz de cambiar un nivel de voltaje a otro. Se usa para proporcionar el nivel correcto de tensión para los muchos componentes eléctricos a bordo del satélite. Se utiliza por ejemplo, para proporcionar el voltaje correcto para el Modulo de Computador a Bordo (The On-Board Computer $\mathrm{OBC}$ ). Los reguladores convertidores dc-dc pueden ser lineales o conmutados.

\subsection{Baterias}

Las baterías seleccionadas son baterías de litio ion polímero por sus múltiples ventajas, entre las que hay que destacar: su menor costo de fabricación, su gran densidad de energía, tasas de descarga superiores, su bajo peso, y volumen, son baterías flexibles que se pueden adaptar a la forma y volumen de un dispositivo en particular, los altos ciclos útiles de trabajo en el espacio exterior; es precisamente por estas características que la batería de litio ion polímero son ampliamente usadas para dispositivos en el mundo de la tecnología de consumo actual.

Entre sus desventajas, están que se necesita un control para la temperatura, puesto que son muy sensibles a esta, y control para el inicio y terminación de carga.

\subsection{Calentador de Baterias}

El picosatélite deberá soportar temperaturas extremas en el exterior que pueden ir desde los $-40^{\circ} \mathrm{C}$ a los $85^{\circ} \mathrm{C}$ como producto de la radiación solar y la radiación infrarroja directamente, y por los momentos de eclipse. Todos cambios térmicos bruscos. Esto repercute en la temperatura interna del picosatélite, que requiere antes que nada un nivel de aislamiento externo adecuado. Pero aun así con un buen aislamiento, al interior del picosatélite las temperaturas podrían variar en márgenes no deseables que puede afectar componentes críticos. A la selección de componentes electrónicos que soporten márgenes de temperatura amplios se debe diseñar un sistema de calefacción interna para protegerlos. Existen dos formas de manejar la parte de control termal: una es mediante un sistema de calefacción pasiva, y la otra mediante un control de calefacción activa. 


\subsection{Regulador Lineal}

Un regulador lineal es un regulador de tensión basado en un dispositivo activo (como un transistor de unión bipolar, transistor de efecto de campo o de tubo de vacío) que operan en su región "lineal" o dispositivos pasivos como diodos Zener operado en su región de avalancha.

Un transistor (u otro dispositivo) se utiliza como un divisor de potencial para controlar la tensión de salida, y un circuito de realimentación compara la tensión de salida a un voltaje de referencia a fin de ajustar la entrada del transistor, lo que mantiene la tensión de salida razonablemente constante. Esto es ineficiente: dado que el transistor actúa como una resistencia, generando un desperdicio de energía eléctrica en forma de calor excesivo. De hecho, la pérdida de potencia debido al calentamiento en el transistor es el valor de la corriente, multiplicado por la caída de tensión en el transistor.

\subsection{Reguladores Conmutados}

Los reguladores conmutados electrónicos DC-DC convierten un nivel de voltaje DC a otro, mediante el almacenamiento temporal de la energía de entrada y luego libera esta energía a la salida con una tensión diferente. El almacenamiento de energía puede ser en componentes de campo magnético (inductores, transformadores) o componentes de campo eléctrico (condensadores). Este método de conversión de energía es más eficiente (75\% a 98\%) que la regulación de voltaje lineal (que disipa energía en forma de calor no deseado). Esta eficiencia es benéfica al incrementar el tiempo de operación de dispositivos que utilizan baterías. La eficiencia se ha incrementado desde finales de 1980 debido a la utilización de FET de potencia, que son capaces de conmutar a una más alta frecuencia que los transistores bipolares, que tienen más pérdidas de conmutación y requieren de circuitos de transmisión más complejos.

Entre las desventajas de los convertidores de conmutación se incluyen la complejidad, el ruido electrónico (EMI / RFI) y, en cierta medida los costos, aunque esto se ha reducido con los avances en el diseño de chips.

Convertidores DC a DC ya están disponibles como circuitos integrados que necesitan un mínimo de componentes adicionales.

La topología básica elegida de la figura 1 comprende 2 conversores dc/dc, uno para la entrada de los paneles solares y el otro para la salida hacia los usuarios.

La figura 6 es un esquema de la configuración elegida para la interconexión de los subsistemas que integran la EPS. El satélite obtiene la energía a partir de un arreglo 
de celdas solares ubicadas en las seis caras del satélite. Existen dos alternativas para la entrada del circuito regulador. Una opción es implementar un circuito seguidor del punto de potencia máximo (MPPT, por sus siglas en inglés) del arreglo solar, lo que permitiría la máxima transferencia de potencia entre el arreglo solar y el regulador de entrada. La otra opción es conectar directamente el arreglo de celdas solares al regulador de entrada, lo que simplifica el diseño, aunque no garantiza la máxima transferencia de potencia.

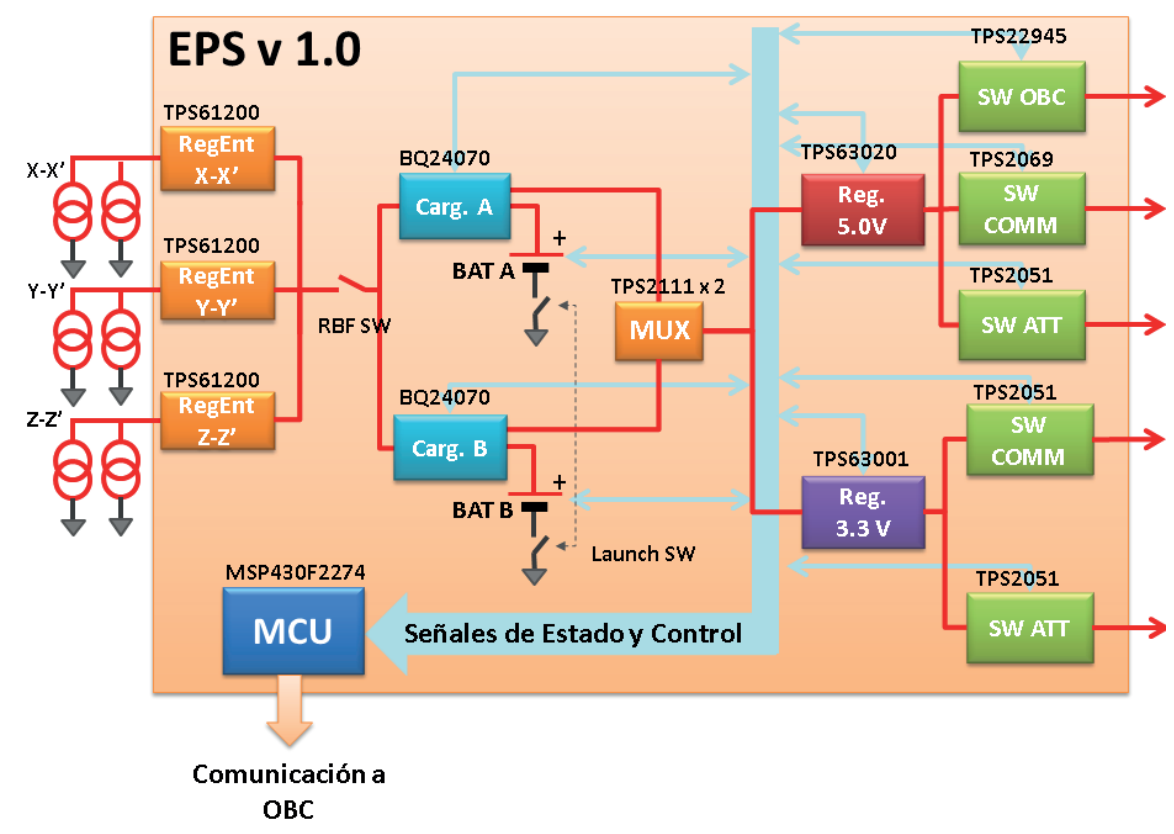

Figura 6: Esquema final elegido de la EPS.

El subsistema de almacenamiento y transferencia de energía tiene como funciones principales el almacenamiento de la energía proveniente de las celdas solares en las baterías. Además, se encarga del monitoreo del estado de las baterías (voltaje, corriente, temperatura, cantidad de carga), y también entrega potencia al sistema bien sea desde los paneles solares y/o desde las baterías cuando los primeros no estén en capacidad de entregar la potencia requerida.

Luego están los reguladores DC-DC de los usuarios, que transforman la potencia en los niveles de voltaje y corriente que requieren los otros módulos del satélite. Así tenemos dos buses de potencia, de 5.0 $\mathrm{V}$ a 1.2A y $3.3 \mathrm{~V}$ a $1.0 \mathrm{~A}$.

Por último, se encuentran el subsistema de distribución y protección a usuarios, encargado de habilitar o deshabilitar usuarios de acuerdo a prioridades establecidas por el OBC. Hay unas señales de estado y control entre los diferentes subsistemas o Módulos y el $\mathrm{OBC}$ que sirven para monitorear y controlar el correcto funcionamiento 
del picosatelite. Así mismo existen señales de control y correcto funcionamiento entre los diferentes bloques de la EPS y su Microcontrolador.

\section{IMPLEMENTACION DE LA EPS}

\subsection{Regulador de entrada}

Su función es elevar el Voltaje que proporcionan los Paneles Solares a un nivel de voltaje adecuado para el Cargador de Baterías. El Circuito Integrado de la Texas Instruments: el TPS61202 es un circuito convertidor elevador-reductor dc-dc conmutado, enfocado al suministro de energía para baterías Alkalinas, de $\mathrm{NiCd}$ o $\mathrm{NiMH}$, de una, dos o tres celdas, o para baterías de Li-Ion o Li-Polímero de una celda, a partir de fuentes de energía de bajo poder como son las celdas solares.

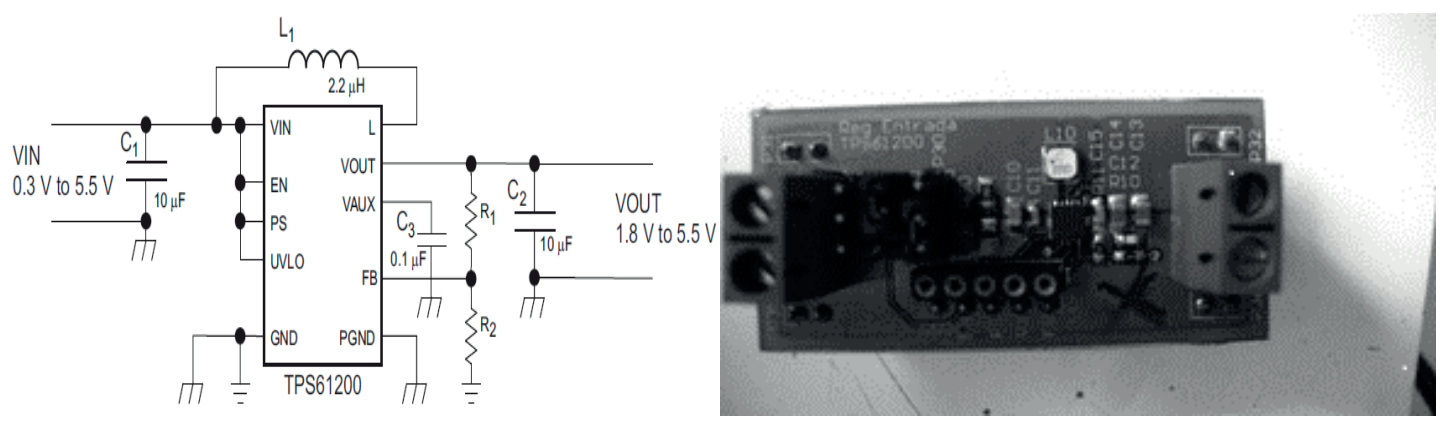

Figura 7: Regulador de Entrada utilizando TPS61202.

Se le puede configurar para que en modo ahorrador de energía cuando hay bajas corrientes bajas de carga.

Todo esto en un encapsulado de $3 \mathrm{~mm}$ por $3 \mathrm{~mm}$.

\subsection{MPPT}

En principio el regulador de entrada contiene un MPPT de punto fijo o variable que permita la máxima transferencia de potencia hacia el cargador de baterías y hacia la salida.

Se comparar el voltaje de entrada con el de salida para ajustar la Conmutación del Regulador de Entrada y así proporcionar la máxima corriente posible sin caídas en el voltaje de salida. 


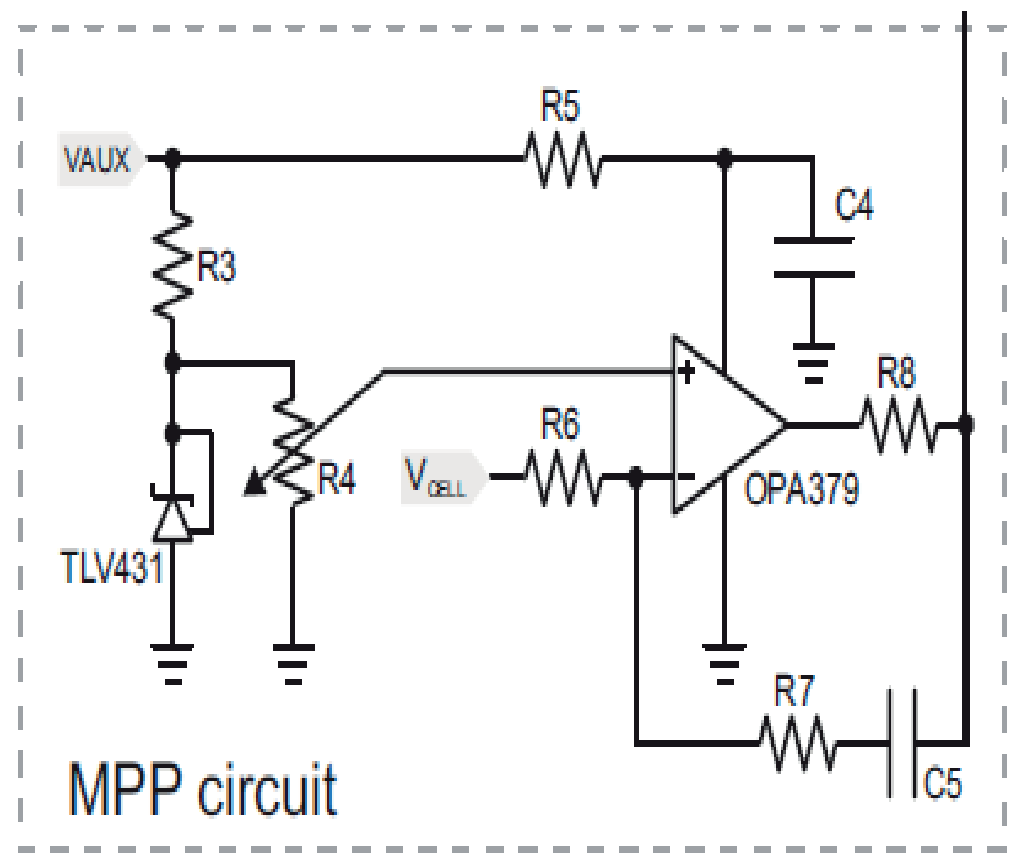

Figura 8: Sistema MPPT.

\subsection{Subsistema de Almacenamiento y Transferencia de Energía}

Su función es gestionar los caminos de energía entré entrada que viene de los Paneles Solares, la Batería y los respectivos buses de alimentación y distribución a usuarios.
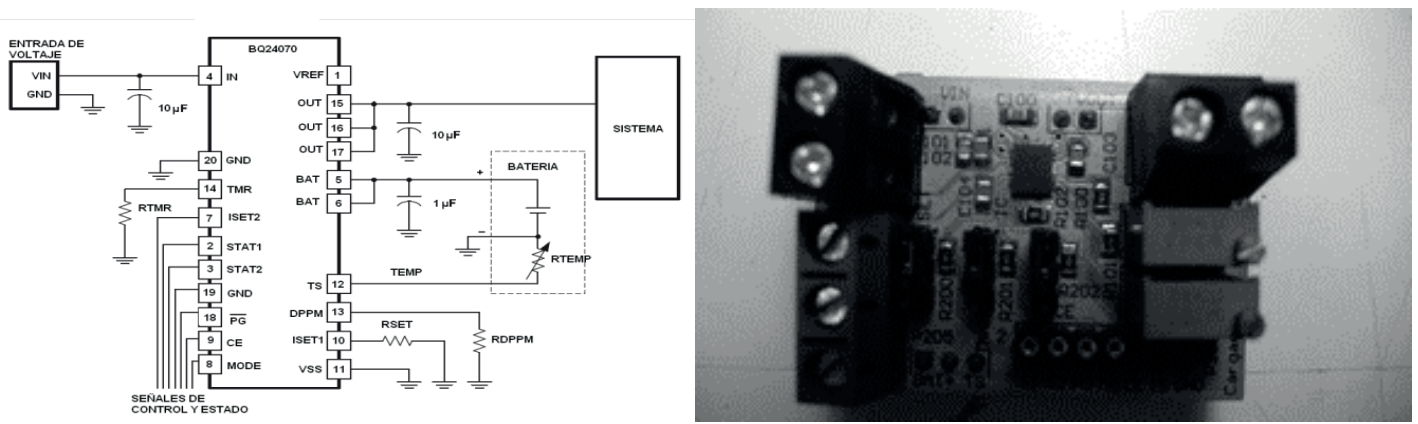

Figura 9: Cargador de Batería bq24070.

Para el circuito de carga de la batería se eligió el BQ24070 del fabricante Texas Instruments que es un cargador lineal altamente integrado para baterías Li-ion, Li-Poly que cuenta con un sistema de administración de camino de potencia enfocado a aplicaciones portátiles de espacio limitado. Algunas características son: pequeño encapsulado QFN de 3,5 mm ×4,5 mm, diseñado para aplicaciones portátiles con baterías de LiIon o Li-Poly de una sola celda, administración de camino de potencia dinámico (DPPM) integrada, ésta característica permite al voltaje de entrada proporcionar energía al 
sistema y cargar la batería simultáneamente. Además, reduce los ciclos de carga y descarga de la batería, permite una terminación de carga adecuada y permite que el sistema funcione con una batería ausente o defectuosa.

\subsection{Protección de Corriente inversa, cortocircuito y térmica.}

Reguladores DC-DC para Buses de Usuarios

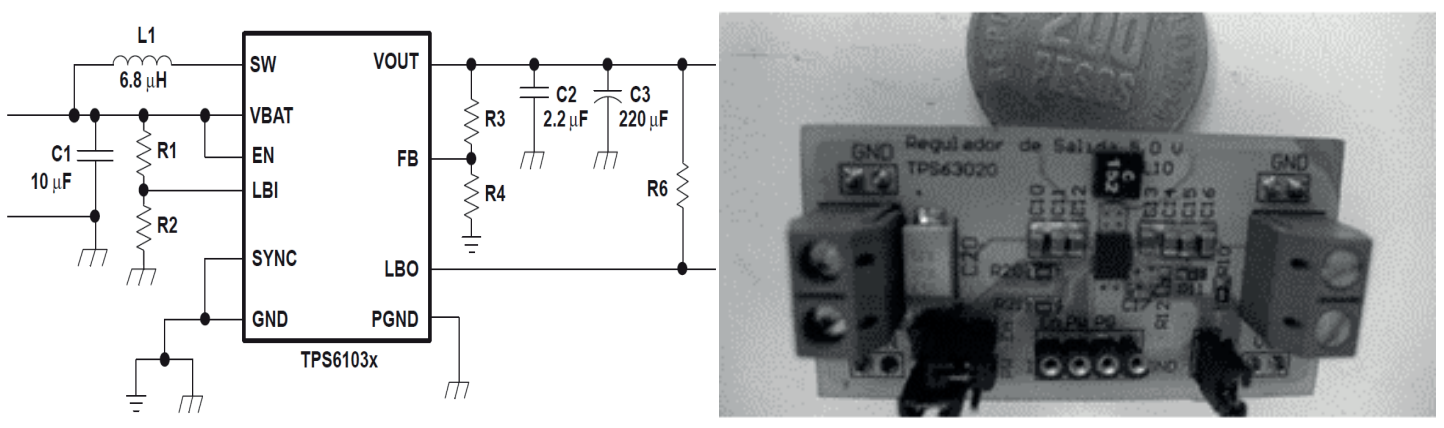

Figura 10: TPS61032 de Texas Instruments para Bus de 5.0V.
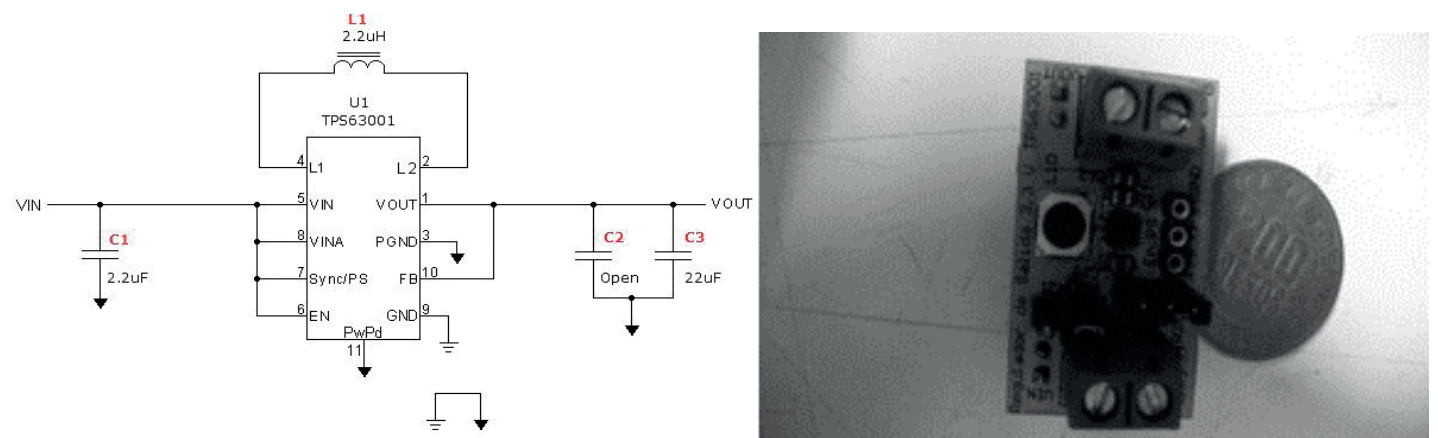

Figura 11: TPS63001 de Texas Instruments para Bus de 3.3V.

El Circuito Integrado de la Texas Instruments TPS63001 proporciona alimentación a buses de usuarios a 3.3V, desde o hacia sistemas basados en baterías alcalinas, de NiCd o NiMH, de una, dos o tres celdas, y a baterías de Li-Ion o Li-polímero de una celda. Tiene corrientes de salida puede pueden ser tan altas como $1.200 \mathrm{~mA}$ mientras se usan baterías de celda simple Li-Ion o Li-polímero, y descargas de hasta $2.5 \mathrm{~V}$ o menos. El funcionamiento del convertidor buck-boost tiene como base una frecuencia fija, y es a través de Modulación por Ancho de Pulso (PWM), y es controlado usando rectificación síncrona para obtener la máxima eficiencia. En cargas de baja corriente, el convertidor entra en modo de ahorro de energía para mantener una alta eficiencia en un amplio rango de corriente de carga. El modo de ahorro de energía puede ser desactivado, 
forzando al convertidor a funcionar a una frecuencia de conmutación fija. El promedio máximo de corriente actual en los interruptores se limita a un valor típico de $1800 \mathrm{~mA}$ la tensión de salida se programa usando un divisor de resistencia externa, o se fija internamente en el chip. El convertidor se puede desactivar para reducir la descarga de la batería. Durante el apagado, la carga se desconecta de la batería. El dispositivo viene en un encapsulado de 10 pines tipo QFN PowerPAD y mide $3 \mathrm{~mm} \times 3 \mathrm{~mm}$ (RDC).

En el caso del Circuito Integrado TPS61032, es un regulador conmutado tipo boost, que proporciona un nivel de bus de 5.0V o ajustable, y es capaz de suministrar corrientes tan altas como $4 \mathrm{~A}$. Todo en un encapsulado de $3 \mathrm{~mm}$ por $4 \mathrm{~mm}$. E igual a la familia TPS6300X, tienen protección contra sobrecorriente y térmica. Tiene limitador de corriente de salida. Hace la transición automática entre el modo reductor, y el modo elevador.

\subsection{Subsistema de Distribución y Protección}
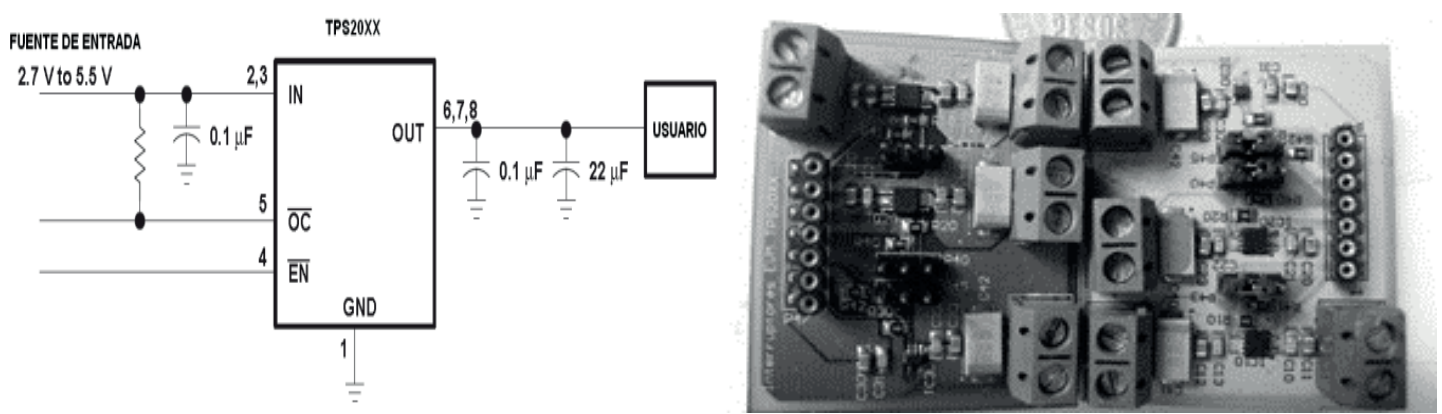

Figura 12: Familia TPS20XX de Texas para Proteccion y Distribucion a usuarios.

Estos dispositivos son conmutadores de distribución de energía y están diseñados para aplicaciones donde es muy probable que presente pesadas cargas capacitivas y/o cortocircuitos. Algunas características de estos conmutadores son: interruptores MOSFET de 7omohms, corriente continua por canal, protección térmica independiente/autónoma contra corto circuito con salida lógica por sobre corriente, rango de funcionamiento de $2.7 \mathrm{~V}$ a 5.5V, entradas compatibles habilitadoras CMOS-TTL, tiempo de subida típica de $2.5 \mathrm{~ms}$, bloqueo por baja tensión, máximo suministro de corriente en modo Standby de 10uA, Interruptor Bidireccional si así se requiere, rango de temperatura desde $0^{\circ} \mathrm{C}$ hasta $85^{\circ} \mathrm{C}$. 


\subsection{Microprocesador}

Toda la programación y la gestión del Módulo se hace a través de un Microprocesador de la Texas Instruments de la familia MSP430. En la figura 13 se aprecia el Microcontrolador.

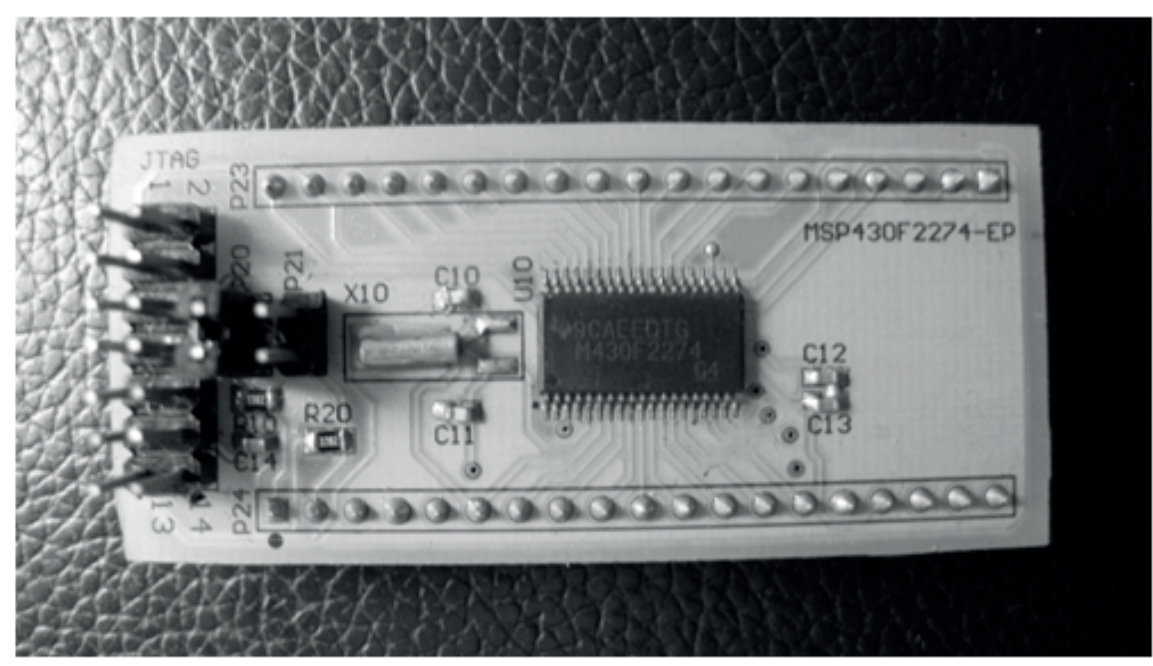

Figura 13: MicroProcesador MSP430.

\subsection{Diseño esquemático y pcb}

PCB: printed circuit board, en español, Tarjeta de Circuito Impreso, el diseño se realizó utilizando la herramienta computacional Altium Designer, para el diseño del esquemático y la PCB.

Cada una de las etapas que conforman el Modulo de Potencia fue diseñado en sus respectivas tarjetas de evaluación, con el fin de hacer las pruebas eléctricas a la circuitería asociada a cada uno de los circuitos integrados. Las fotos de las tarjetas se observan en las figuras 7 a 13. Estas Tarjetas de Evaluación representan la implementación de los diferentes bloques del Módulo de Potencia.

Hechas las pruebas a las tarjetas de Evaluación el paso siguiente fue la fabricación, y ensamble de la tarjeta final, el Modulo de la EPS versión 1.0, tal como se muestra en la figura 14 .

No fue necesario implementar un sistema de MPPT ya que los circuitos empleados ajustan los niveles de potencia para máxima eficiencia. 

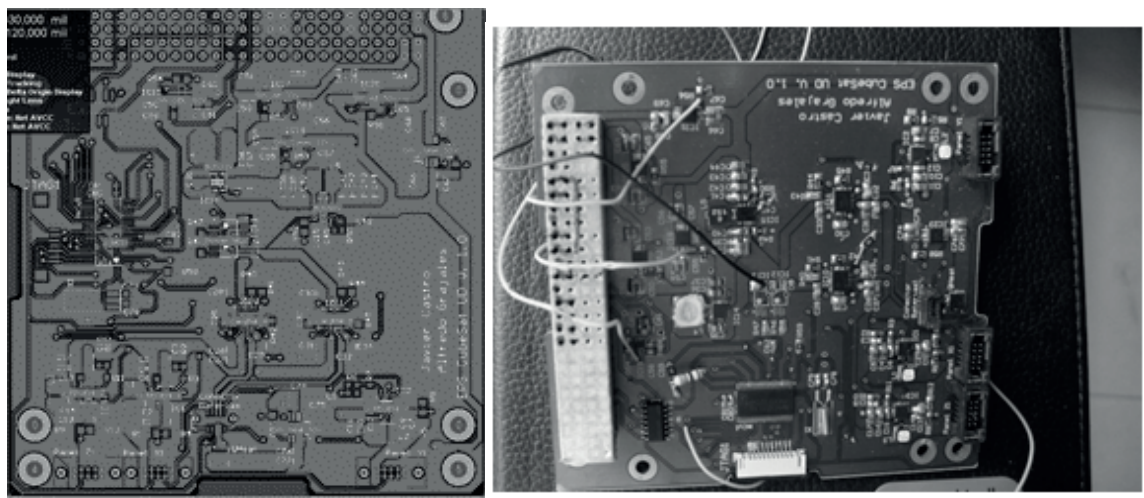

Figura 14: EPS v1.o Diseño Altium

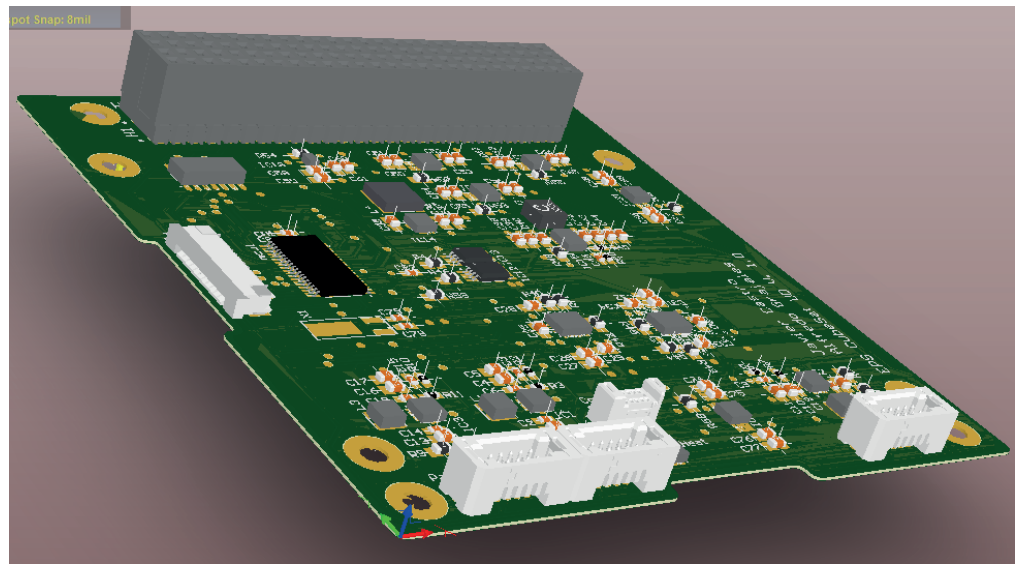

Figura 15: Vista en 3D del Módulo de Potencia Versión 1 diseñado con Altium Designer.

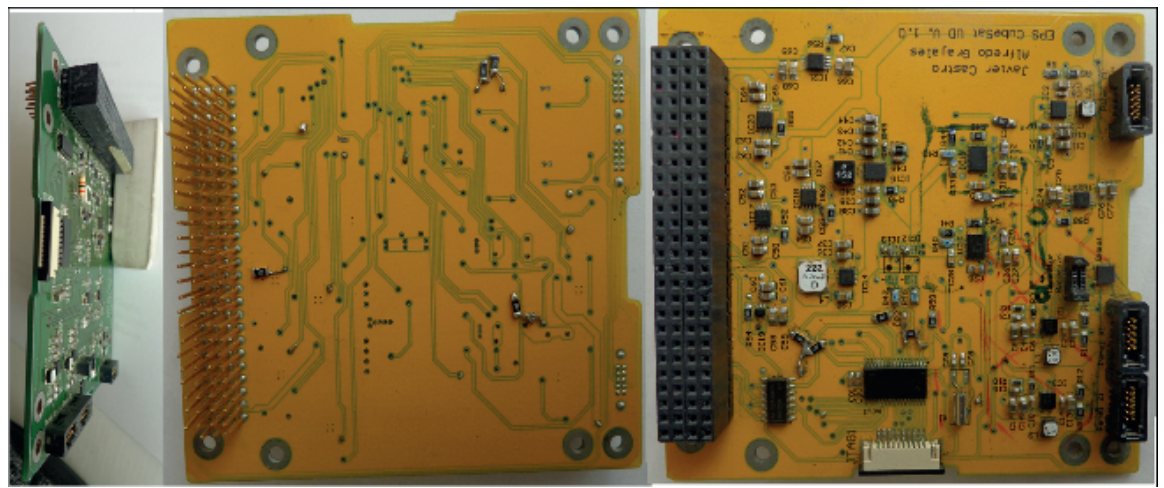

Figura 16: EPS v1.0

\section{RESULTADOS Y CONLUSIONES}

El tipo de conversores utilizados en los reguladores DC/DC son del tipo conmutado tipo elevador o elevador-reductor con eficiencias mayores al 90\%, verificadas en las pruebas. 
El Cargador de baterías del tipo lineal permite la administración del Camino de Potencia Dinámico, esto es le da prioridad a los paneles solares siempre como fuente principal, mientras se distribuye energía a los sistemas de usuarios como prioridad y carga la batería al mismo tiempo. También si los usuarios lo demandan se puede tomar energía tanto de los paneles solares como de las baterías.

Se utilizó para cada usuario un sistema de autoprotección al usuario contra sobrecorriente, sobrevoltaje y temperaturas elevadas, limitando la corriente o suprimiéndola, e indicando un estado de falla.

Para darle mayor robustez al satélite se han implementado tres reguladores de entrada por cada par de paneles opuestos colocados en paralelo, al mismo tiempo, esto permite obtener datos de telemetría si así se dispone.

Las pruebas eléctricas hechas a esta tarjeta, la EPS v1.0 se hicieron manualmente sin control de microprocesador, los resultados obtenidos se asemejan muy bien con el comportamiento esperado de acuerdo a las hojas de especificaciones de los componentes.

El Modulo de la EPS para la versión 2.0 que está en proceso de implementación incorpora la programación para controlar y auto gestionar el picosatelite, y para la Comunicación entre bloques de la EPS y subsistemas del picosatelite.

\section{Referencias}

[1] Duan, L., Loh, J.T., and Chen, W.F. (1990). "M-P-F based analysis of dented tubular members". Journal of Structural Engineering, Vol. 21, No. 8, pp 34-44.

[2] CHAVES GARCIA, Augusto E. Sistema de Potencia Fotovoltaica para Equipos Remotos: Diseño del Módulo de Potencia Picosatélite Experimental CubeSAT UD. Bogotá, 2008, 137 h. Trabajo de grado (Ingeniero Electrónico). Universidad Distrital Francisco José de Caldas. Facultad de Ingeniería. Proyecto Curricular de Ingeniería Electrónica.

[3] DAY, Christopher Alan. The Design of an Efficient, Elegant, and Cubic Pico-Satellite Electronics System. San Luis Obispo, 2004, 108 h. Thesis (Master of Science in Electrical Engineering). California Polytechnic State University.

[4] LARSON, Wiley J.; WERTZ James R. Space Mission Analysis and Design. El Segundo CA: Microcosm Press and Kluwer Academic Publishers, 1999, 987 p. ISBN 1-881883$10-8$.

[5] ALMINDE, Lars; BISGAARD, Morten. Design of Hardware and Software for the Powersupply for AAU Cubesat. Aalburg, 2002, 39 h. Reporte Group 
02gr733. Institute of Electronic Systems. University of Aalborg. Disponible en: http://www.cubesat.auc.dk/documents/psu/new_psu.pdf

[6] ALMINDE, Lars; BISGAARD, Morten. Power Supply for the AAU Cubesat. Aalburg, 2001, 239 h. Reporte Group 019r509. Institute of Electronic Systems. University of Aalborg. Disponible en: http://www.cubesat.auc.dk/dokumenter/psu.pdf

[7] DAN LAZAR, Radu; BUCELEA, Vasile. Optimized Design of Power Supply for CubeSat at Aalborg University. Aalburg, 2001, 163 h. Reporte GROUP PED9-17C. Institute of Energy Technology. Aalborg University. Disponible en: http://www.cubesat.auc.dk/psu.html

[8] DORN, Lawrence Tyrone Jr. Nps-Scat; Electrical Power System. Monterrey CA. 2009, 103 h. Master's Thesis. Naval Postgraduate School. Disponible en: http://www.dtic.mil/cgibin/GetTRDoc? Location=U2\&doc $=$ GetTRDoc.pdf\&AD=ADA508860

[9] KRISHNAMURTHY, Narayanan. Dynamic Modelling of CubeSat Project MOVE. Luleå, Sweden, 2008, 129 h. Master Thesis, Continuation Courses Space Science and Technology. Department of Space Science, Kiruna. Luleå University of Technology. Disponible en: http://epubl.Itu.se/1653-0187/2008/080/LTU-PB-EX-08080-SE.pdf

[10] CHAN, Roger; BENERJEE, Rajib; JANI, Anang. Win-Cube Project: Electrical Power System Phase Two Critical Design Review. Winnipeg, Canada, 2008, 152 h. Final Report (Bachelor of Science in Electrical Engineering). Faculty of Engineering. University of Manitoba. Disponible en: http://Itc.umanitoba.ca/wincube/images/a/ab/Final_Report_1.pdf

\section{Authorization and Disclaimer}

Authors authorize ESTEC to publish the paper in the conference proceedings. Neither ESTEC nor the editors are responsible either for the content or for the implications of what is expressed in the paper. 